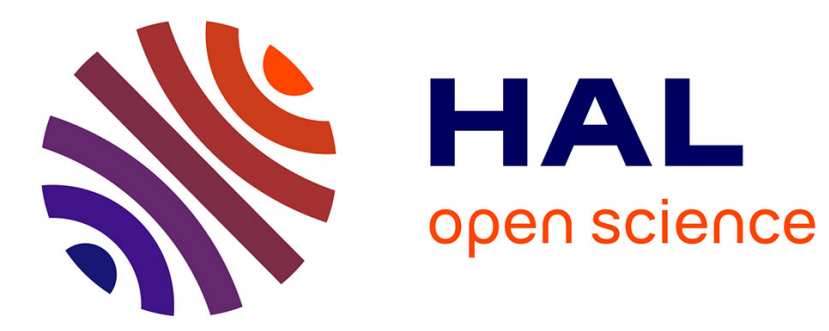

\title{
Microscopic description of nuclear fission
}

\author{
J.-F. Berger, M. Girod, D. Gogny
}

\section{To cite this version:}

J.-F. Berger, M. Girod, D. Gogny. Microscopic description of nuclear fission. Journal de Physique Lettres, 1981, 42 (23), pp.509-511. 10.1051/jphyslet:019810042023050900 . jpa-00231988

\section{HAL Id: jpa-00231988 https://hal.science/jpa-00231988}

Submitted on 1 Jan 1981

HAL is a multi-disciplinary open access archive for the deposit and dissemination of scientific research documents, whether they are published or not. The documents may come from teaching and research institutions in France or abroad, or from public or private research centers.
L'archive ouverte pluridisciplinaire HAL, est destinée au dépôt et à la diffusion de documents scientifiques de niveau recherche, publiés ou non, émanant des établissements d'enseignement et de recherche français ou étrangers, des laboratoires publics ou privés. 


\title{
LE JOURNAL DE PHYSIQUE-LETTRES
}

J. Physique - LETTRES 42 (1981) L-509 - L-511

1er DÉCEMBRE 1981, PAGE L-509

Classification

Physics Abstracts

$21.60-24.75$

\section{Microscopic description of nuclear fission $\left({ }^{*}\right)$}

\author{
J.-F. Berger, M. Girod and D. Gogny \\ Service de Physique Neutronique et Nucléaire, Centre d'Etudes de Bruyères-le-Châtel, \\ B.P. n ${ }^{\circ}$ 561, 92542 Montrouge Cedex, France
}

(Reçu le 28 septembre 1981, accepté le 13 octobre 1981)

\begin{abstract}
Résumé. - Partant d'une description complètement microscopique du noyau, nous tentons d'interpréter la fission du noyau ${ }^{240} \mathrm{Pu}$ en termes de coordonnées collectives considérées comme variables dynamiques. Nous insistons en particulier sur le mécanisme de la scission.

Abstract. - Starting from a completely microscopic description of the nucleus, we try to extract the characteristic features of the collective dynamics of the fission phenomenon in the case of the ${ }^{240} \mathrm{Pu}$ nucleus. In particular, we give an interpretation for the mechanism of the scission process.
\end{abstract}

The fission process can be viewed as resulting from the interplay of two kinds of nuclear motions. The first is the coherent motion of the nucleons in bulk which forces the nucleus to stretch and eventually break into two fragments. The second is the internal motion of the nucleons in the nuclear field of the fissioning system. Of course, these two kinds of motion are not independent. In particular, the internal structure of the nucleus strongly influences the dynamics of the coherent motion.

In the case of low energy binary fission, the coherent motion of the nucleus can be described by means of a few collective parameters $\left(q_{1}, q_{2}, \ldots\right)$ characterizing the shape of the system. The most important of them are those measuring the total stretching of the nucleus, its left-right asymmetry and its axial asymmetry. Besides, the characteristic time for the coherent motion $\left(\sim 10^{-21} \mathrm{~s}\right)$ is far greater than that associated with the internal motion of the nucleons $\left(\sim 10^{-22} \mathrm{~s}\right)$. Consequently, the internal structure of the nucleus can be assumed to be in equilibrium at each step of the collective motion. The internal degrees of freedom of the system can therefore be

(*) La version française de cet article a été proposée pour publication aux Comptes Rendus de l'Académie des Sciences. described using the adiabatic approximation. In this case, the fission properties of the nucleus depend mainly on the so-called potential energy surface $E=V\left(q_{1}, q_{2}, \ldots\right)$ of the system. This function represents the energy to be supplied to the system in order that its deformation parameters become $\left(q_{1}, q_{2}, \ldots\right)$.

Two approaches have been developed in recent years to compute these nuclear potential energy surfaces. The first one has been proposed by Strutinsky [1]. It consists of adding to the deformation energy of a classical charged liquid drop a phenomenological correction which simulates the quantum effects existing in the internal structure of the nucleus (the shell effects). The second method is based on selfconsistent approaches of the Hartree-Fock type. It places the A nucleons of the system in one or more external deforming fields. The total energy of the system is then computed in a completely microscopic way. The latter method is favoured for two reasons. First, the only parameters of the theory are those of the nuclear interaction. Second, the collective effects (liquid-drop effects) are treated on the same footing as the internal effects (shell effects, pairing correlations).

We present in the following some results obtained in such a self-consistent microscopic approach for 
the case of the ${ }^{240} \mathrm{Pu}$ nucleus. As compared with previous works [2] our approach includes several improvements :

1) We use an effective nuclear force whose properties (range, saturation properties, compressibility, ...) are particularly realistic [3].

2) Our microscopic method is the extension of the mean field theory which allows a completely self-consistent treatment of the pairing effects (HartreeFock-Bogolyubov (HFB) theory).

3) Besides the total deformation of the system, our description takes into account the possibility of leftright an non-axial asymmetric deformations. As external fields we have chosen the multipolar operators

$$
\begin{aligned}
& \hat{Q}_{20}=\sqrt{16 \pi} / 5 \sum_{i}\left(r_{i}\right)^{2} Y_{2}^{0} \\
& \hat{Q}_{30}=\sqrt{4 \pi} / 7 \sum_{i}\left(r_{i}\right)^{3} Y_{3}^{0}
\end{aligned}
$$

and

$$
\hat{Q}_{22}=\sqrt{8 \pi} / 15 \sum_{i}\left(r_{i}\right)^{2}\left(Y_{2}^{2}+Y_{2}^{-2}\right)
$$

respectively. The collective variables are the expectation values of the corresponding operators.

4) Finally, being expectation values of operators, our collective variables undergo fluctuations. Consequently, we have included the zero-point energies associated with these fluctuations in our potential energy surfaces. In this way, the surfaces effectively represent the potential to be used in a semi-classical description of collective motion.

The two-dimensional potential energy surface $E=U\left(\left\langle\hat{Q}_{20}\right\rangle,\left\langle\hat{Q}_{30}\right\rangle\right)$ is shown on figure 1. The two minima $P_{1}\left(\left\langle\hat{Q}_{20}\right\rangle \sim 28 \mathrm{~b},\left\langle\hat{Q}_{30}\right\rangle \sim 0\right)$ and $\mathrm{P}_{2}\left(\left\langle\hat{Q}_{20}^{\prime}\right\rangle \sim 92 \mathrm{~b},\left\langle\hat{Q}_{30}\right\rangle \sim 0\right)$ indicate the equilibrium deformations of the ground state and isomeric

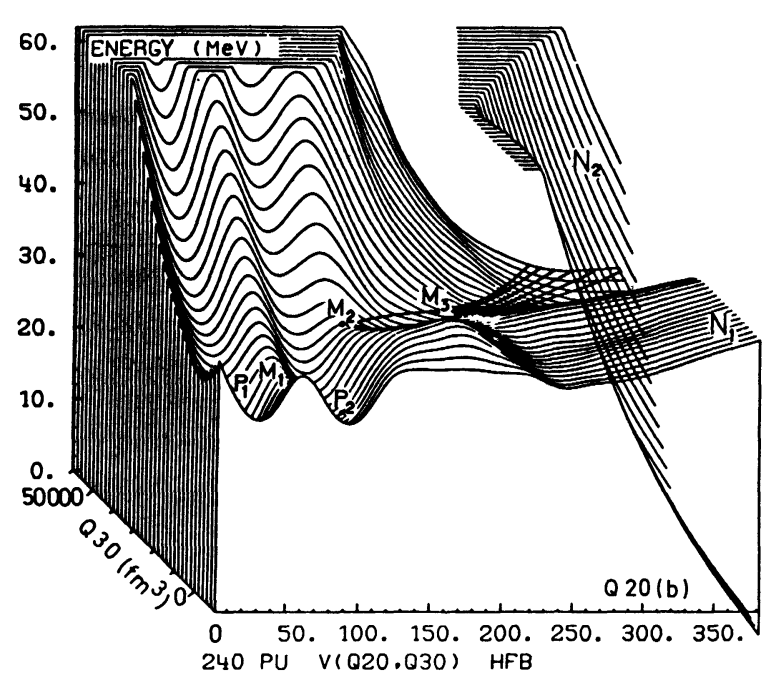

Fig. 1. - Potential energy surface of the ${ }^{240} \mathrm{Pu}$ nucleus with respect to the collective variables $\left\langle\hat{Q}_{20}\right\rangle$ and $\left\langle\hat{Q}_{30}\right\rangle$. state of ${ }^{240} \mathrm{Pu}$. These deformations are in excellent agreement with the values deduced from experiment. Two saddle points $\mathbf{M}_{1}$ and $\mathbf{M}_{2}$ have also been represented on the surface. Our results show that the more stable configuration of the nucleus is non-axial in $M_{1}$ and more and more left-right asymmetric beyond $\mathbf{M}_{2}$. The most probable asymmetry in the scission region is quite compatible with the experimental mass distribution of ${ }^{240} \mathrm{Pu}$ fission fragments.

For larger deformations $\left.\left(\left\langle\hat{Q}_{20}\right\rangle\right\rangle 230 \mathrm{~b}\right)$ a new feature appears : for each pair of parameters $\left(\left\langle\hat{Q}_{20}\right\rangle,\left\langle\hat{Q}_{30}\right\rangle\right)$ the system can be found not only with the shape of a single nucleus, but also as two separate fragments. Then, the potential energy surface contains, apart from the $\mathrm{N}_{1}$ sheet, a second sheet $\mathrm{N}_{2}$ which is completely distinct from the first. Therefore, the transition from non-fragmented to fragmented configuration, in particular the scission of the nucleus, cannot be achieved on the surface of figure 1. In fact, a careful comparison of the nucleon distributions corresponding to the two sheets $N_{1}$ and $\mathrm{N}_{2}$ suggested the introduction of the additional collective variable $\left\langle\hat{Q}_{40}\right\rangle$, where

$$
\hat{Q}_{40}=\sqrt{\frac{4 \pi}{9}} \sum_{i}\left(r_{i}\right)^{4} Y_{4}^{0}
$$

is the hexadecapole operator, in order to describe such a transition. Then, the potential energy surface in the scission region is represented by a function of the three independent variables $\left\langle\hat{Q}_{20}\right\rangle,\left\langle\hat{Q}_{30}\right\rangle$ and $\left\langle\hat{Q}_{40}\right\rangle$. It is described by only one sheet. Figure 2 shows a two-dimensional cut in this surface obtained by constraining the two operators $\hat{Q}_{20}$ and $\hat{Q}_{40}$, while leaving the left-right asymmetry being free. The valleys $\left(V_{1}\right)$ and $\left(V_{2}\right)$ correspond to non-fragmented and fragmented configurations, respectively. The bottoms of valleys $\left(V_{1}\right)$ and $\left(V_{2}\right)$ are curves belonging to the sheets $\left(\mathrm{N}_{1}\right)$ and $\left(\mathrm{N}_{2}\right)$ of figure 1 , respectively, and the scission of the nucleus happens near the top of the barrier between the two valleys.

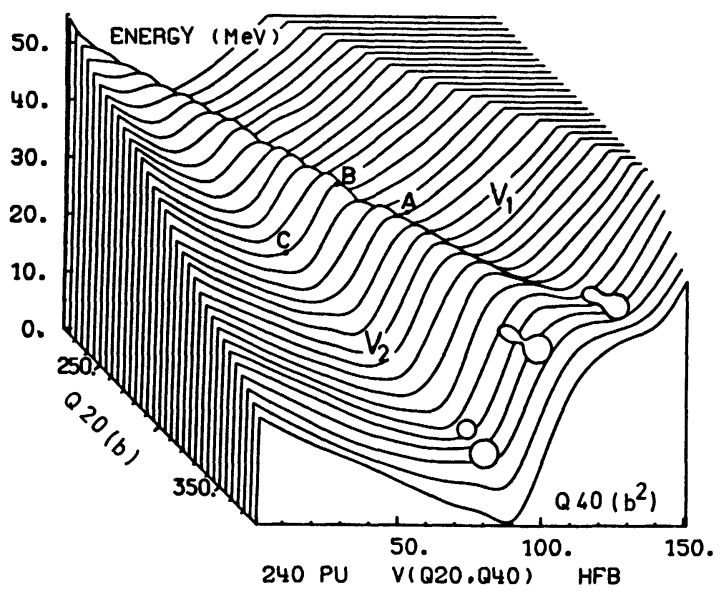

Fig. 2. - Potential energy surface of the ${ }^{240} \mathrm{Pu}$ nucleus with respect to the collective variables $\left\langle\hat{Q}_{20}\right\rangle$ and $\left\langle\hat{Q}_{40}\right\rangle$, in the scission region. 
Figure 3 displays, for instance, the isodensity lines of the system when passing from $\mathrm{A}$ to $\mathrm{C}$ via $\mathrm{B}$ as indicated on figure 2. Note that at deformation $\left\langle\hat{Q}_{20}\right\rangle$ larger than $370 \mathrm{~b}$ the "scission" barrier disappears, and the two valleys become the single valley $\left(\mathrm{V}_{2}\right)$.

We now discuss qualitatively the evolution of the nucleus towards scission along trajectories in the space of the collective coordinates, with these coordinates considered as dynamical variables. Without going into details, we note that this discussion could be placed on a firmer basis by generalizing the Feynman integral to the functional space of the HFB solutions generated in the constrained calculations described above. Also we shall note that the paths followed by the system depend mainly on the potential energy surface. In other words, we shall not take
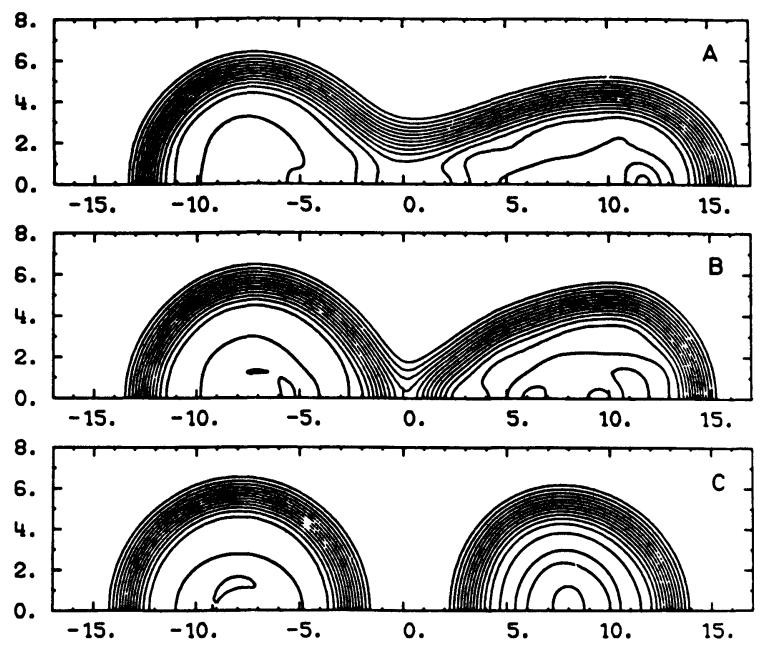

Fig. 3. - Nuclear isodensity curves at the points A, B and C represented in figure 2. The horizontal axis is a symmetry axis for the nucleus, and the lengths are expressed in fermis. into account a possible variation of the nuclear inertia with the collective coordinates. Lastly, the energy is chosen near the fission threshold $(8-10 \mathrm{MeV})$.

In figure 1 have been drawn the most probable paths starting from the saddle point $\mathbf{M}_{2}$. Between $M_{2}$ and $M_{3}$, the path follows the bottom of the valley $\left(\left\langle\hat{Q}_{30}\right\rangle\right.$ is free). Only one path was drawn in order to show that the nucleus is very rigid against $\left\langle\hat{Q}_{30}\right\rangle$. Beyond $\mathbf{M}_{3}$, the valley gets broader as the shaded area indicates and, consequently, one can no longer define a unique path. Various ones can be followed foreshowing different mass fragmentations of the system.

In order to describe the scission, note that we have selected among these paths the one for which the left-right asymmetry constraint was released. We then studied the stability of the system against hexadecapole deformations. Figure 2 suggests the following interpretation : the nucleus stretches in the $\left(V_{1}\right)$ valley until the scission barrier becomes practically negligible $\left(\left\langle\hat{Q}_{20}\right\rangle \sim 370 \mathrm{~b}\right)$. In this case the nucleus follows a trajectory that favours a decreasing of the $\left\langle\hat{Q}_{40}\right\rangle$ variable, eventually ending by the scission into two fragments. The slow variation of the potential energy surface during this evolutive phase justifies the adiabatic assumption on which our interpretation of the scission process is based. Immediately after the scission, the fragments have an internal energy coming from collective excitations. The latter are completely decoupled from the motion of the centres of mass of the fragments which are moving apart under the influence of the Coulomb repulsion.

This discussion is very schematic and it does not exclude less-probable events like scissions occurring from tunnelling all along the scission barrier. These events can lead to fragments having very little excitation energy as observed in the " cold fission " phenomenon induced by thermal neutrons on uranium nuclei [4].

\section{References}

[1] Strutinsky, V. M., Nucl. Phys. A 95 (1967) 420; A 122 (1968) 1

[2] Flocard, H., Quentin, P., Vautherin, D., VÉnÉroni, M. and Kerman, A. K., Nucl. Phys. A 231 (1974) 176

Kolb, D., Cusson, R. Y. and Schmitt, H. W., Phys. Rev. C 10 (1974) 1529.

[3] Dechargé, J. and Gogny, D., Phys. Rev. C 21 (1980) 1568.

[4] Signarbieux, C., Montoya, M., Ribrag, M., Mazur, C., Guet, C., Perrin, P., Maurel, N. M., J. Physique-Lett. 42 (1981) L-437. 